\title{
Role of Multivalent Interactions in Dynamic-Template-Directed Assembly of Conjugated Polymers
}

Erfan Mohammadi ${ }^{1}$, Prapti Kafle ${ }^{1}$, Kai-Yu Huang ${ }^{1}$, Weikun Zhu ${ }^{1}$, Jennifer Huang ${ }^{2}$, Seok-Heon Jung $^{3}$, Jin-Kyun Lee ${ }^{3}$, Christopher M. Evans ${ }^{2}$, and Ying Diao ${ }^{1 *}$

IDepartment of Chemical and Biomolecular Engineering, University of Illinois at Urbana-Champaign, Urbana, Illinois 61801, United States.

${ }^{2}$ Department of Polymer Science \& Engineering, Inha University, Incheon 402-751, South Korea.

${ }^{3}$ Department of Materials Science and Engineering, University of Illinois at Urbana-Champaign, Urbana, Illinois 61801, United States.

*E-mail: yingdiao@illinois.edu 


\section{Supporting information}

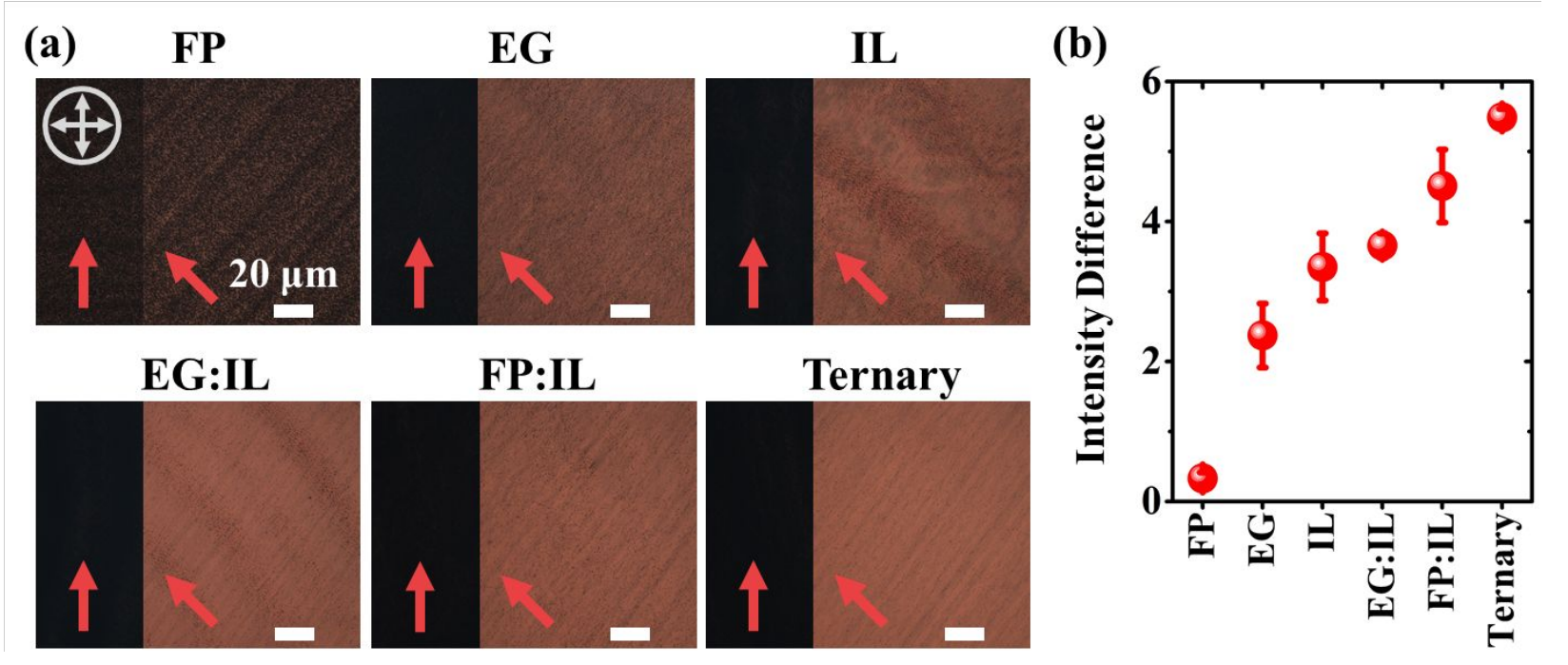

Figure S1. DPP-BTz thin film mesoscale morphology analysis via C-POM. (a) C-POM images with the coating directions (single arrows) parallel and 45 degrees with respect to the cross-polarized light. The orientation of crossed polarizers is denoted as crossed arrows. All scale bars are $20 \mu \mathrm{m}$. With increasing diversity of the dynamic templates, stronger birefringence effect is observed in DPP-BTz thin films. Upon rotation, the whole C-POM image uniformly varies in intensity which indicates that the film is highly ordered at the mesoscale. (b) The normalized average intensity difference as a function of template composition. Larger values correspond to higher birefringence of the polymer film. Intensity difference is independent of thickness since it is normalized by I0. We avoided the cracks in the image analysis. 
(a)

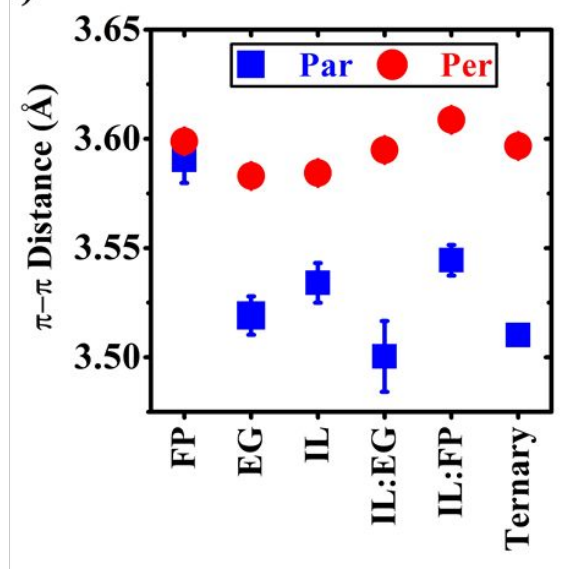

(b)

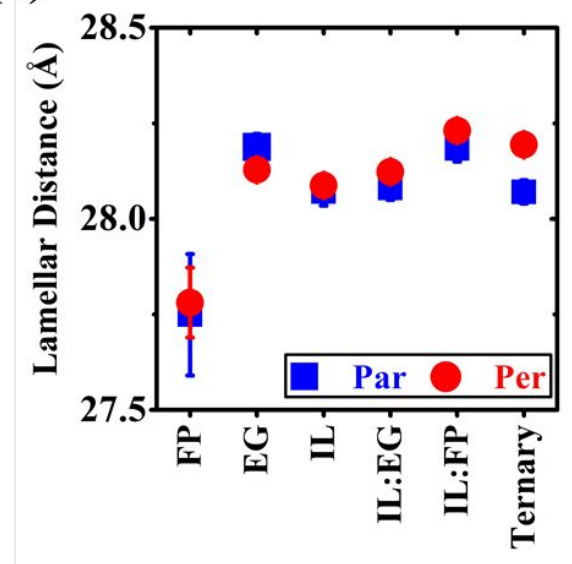

Figure S2. GIXD peak analysis of template-composition-dependent GIXD. (a) $\pi-\pi$ and (b) lamellar stacking distances extracted from (010) and (200) peaks, respectively. $\pi-\pi$ (lamellar) stacking peak represent in-plane (out-of-plane) crystallite packing. For details of GIXD data analysis, please see Methods section in the main text. 


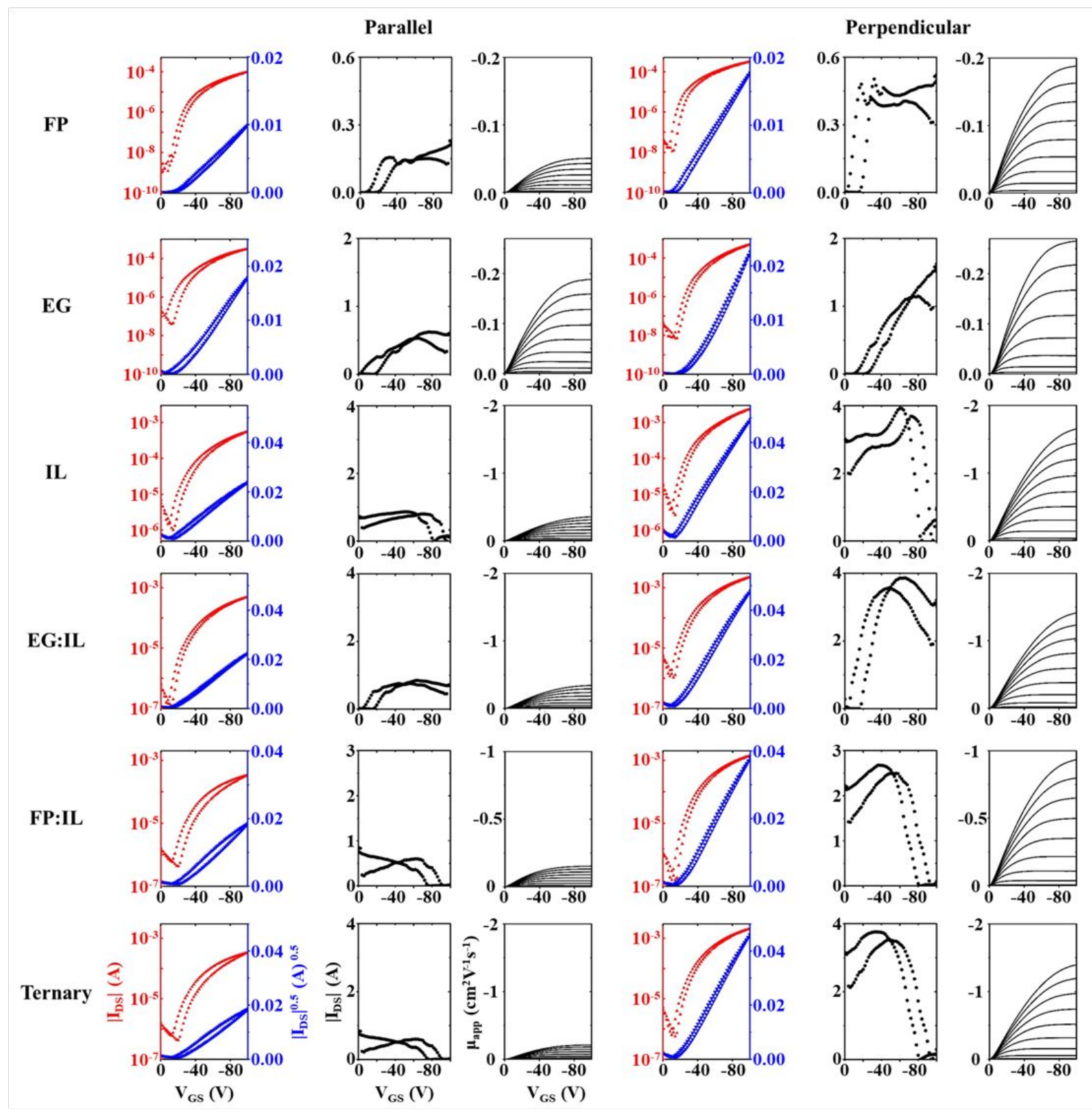

Figure S3. DPP-BTz thin film field-effect transistor output characteristics. Representative transfer, $\mu_{\text {app }}$ vs. $\mathrm{V}_{\mathrm{GS}}$ and output curves for devices fabricated on various substrates. DPP-BTz films were transferred to ODTS-treated $\mathrm{SiO} 2$ and BGTC structure was fabricated by thermally depositing MoO3/Ag electrodes atop $(\mathrm{W}=840 \pm 15 \mu \mathrm{m}, \mathrm{L}=47 \pm 2 \mu \mathrm{m})$. 


\begin{tabular}{|c|c|c|c|c|c|c|}
\hline \multirow{2}{*}{ Template } & \multicolumn{4}{|c|}{ Parallel } & \multicolumn{3}{c|}{ Perpendicular } \\
\cline { 2 - 7 } & $\begin{array}{c}\boldsymbol{\mu}_{\text {app }}(\mathbf{m a x}) \\
\left(\mathbf{c m}^{\mathbf{2}} \mathbf{V}^{-\mathbf{1}} \mathbf{s}^{-1}\right)\end{array}$ & $\begin{array}{c}\mathbf{L o g} \\
\left(\mathbf{I}_{\text {on }} / \mathbf{I}_{\text {off }}\right)\end{array}$ & $\mathbf{V}_{\text {th }} \mathbf{( V )}$ & $\begin{array}{c}\boldsymbol{\mu}_{\text {app }}(\mathbf{m a x}) \\
\left(\mathbf{c m}^{\mathbf{2}} \mathbf{V}^{-1} \mathbf{s}^{-1}\right)\end{array}$ & $\begin{array}{c}\mathbf{L o g} \\
\left(\mathbf{I}_{\text {on }} / \mathbf{I}_{\text {off }}\right)\end{array}$ & $\mathbf{V}_{\text {th }} \mathbf{( V )}$ \\
\hline FP & $0.15 \pm 0.01(0.18)$ & $4.6 \pm 0.7$ & $-9.4 \pm 2.3$ & $0.30 \pm 0.03(0.48)$ & $5.2 \pm 0.7$ & $-16.0 \pm 2.4$ \\
\hline EG & $0.34 \pm 0.06(0.57)$ & $4.9 \pm 0.7$ & $-17.1 \pm 2.0$ & $0.85 \pm 0.06(1.15)$ & $5.3 \pm 0.8$ & $-17.5 \pm 0.6$ \\
\hline IL & $0.76 \pm 0.02(0.87)$ & $2.8 \pm 0.3$ & $-4.8 \pm 1.4$ & $2.45 \pm 0.26(3.61)$ & $4.2 \pm 1.0$ & $-13.9 \pm 2.0$ \\
\hline EG:IL & $0.71 \pm 0.01(0.76)$ & $3.2 \pm 0.2$ & $-9.1 \pm 1.1$ & $2.47 \pm 0.20(3.56)$ & $3.4 \pm 0.2$ & $-17.4 \pm 1.0$ \\
\hline FP:IL & $0.53 \pm 0.03(0.68)$ & $3.7 \pm 0.4$ & $-16.4 \pm 1.0$ & $2.17 \pm 0.26(3.03)$ & $4.5 \pm 0.4$ & $-18.5 \pm 0.8$ \\
\hline Ternary & $0.50 \pm 0.04(0.69)$ & $3.6 \pm 0.2$ & $-14.4 \pm 1.2$ & $2.75 \pm 0.11(3.26)$ & $5.6 \pm 1.5$ & $-18.5 \pm 0.7$ \\
\hline
\end{tabular}

Table S1. Summary of the key FET parameters for DPP-BTz devices. 


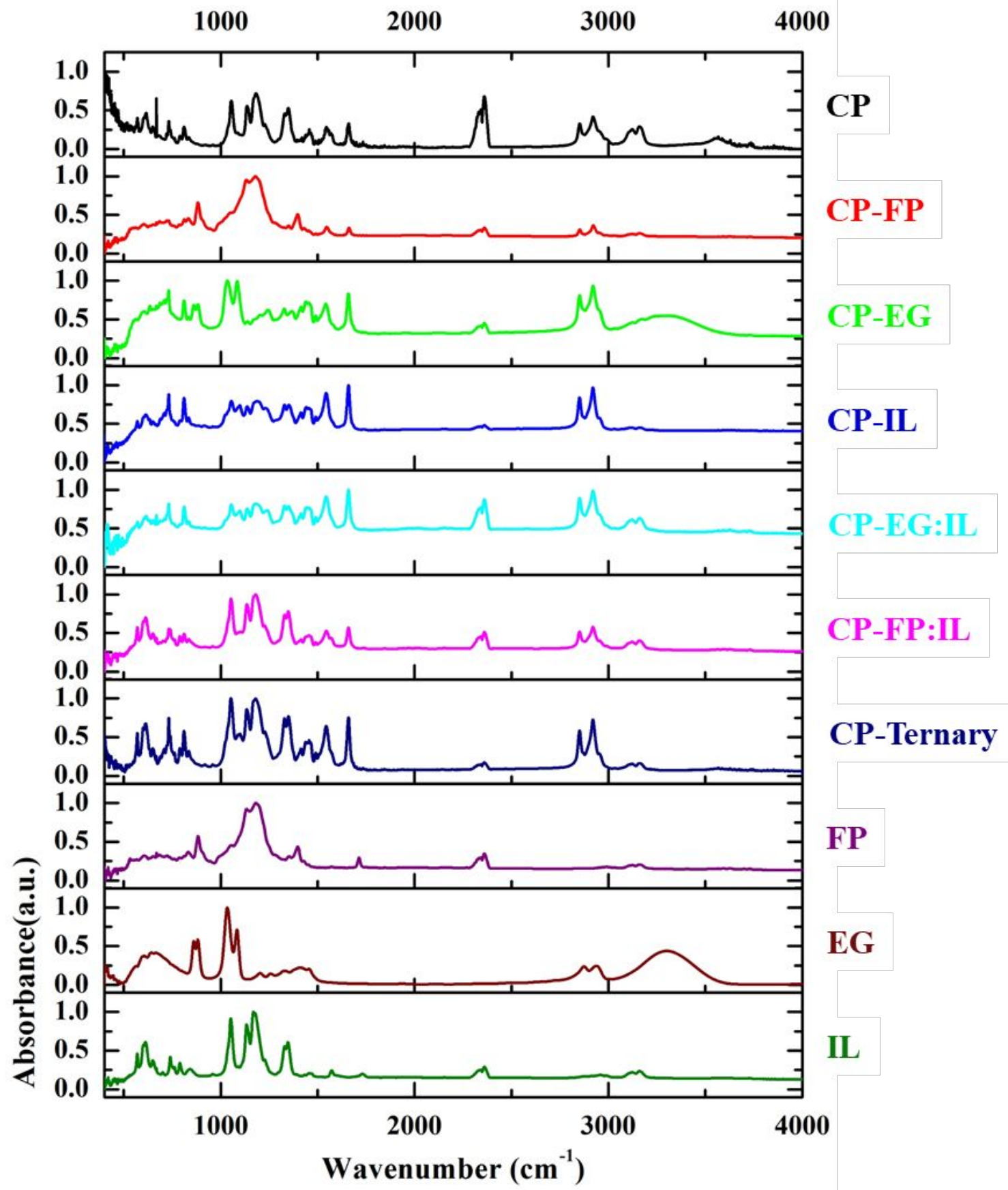

Figure S4. Normalized ATR-FTIR absorbance spectroscopy measurements. The ATR-FTIR spectra were recorded within $400-4000 \mathrm{~cm}^{-1}$ for DPP-BTz with and without templates, and for neat FP, EG and IL. 
(a)

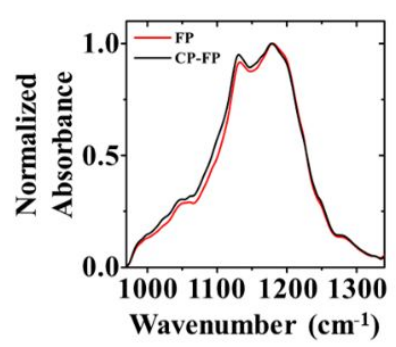

(c)

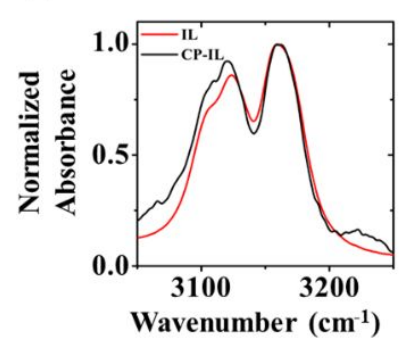

FP F-C-F stretch

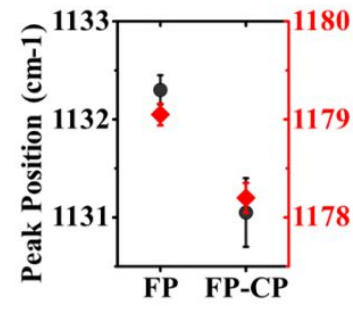

idazole stretch

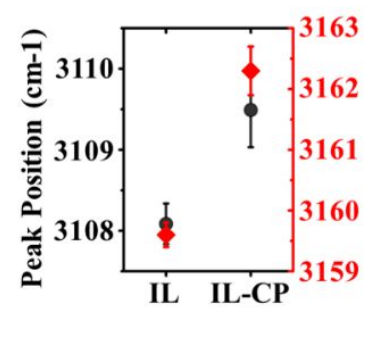

(b)

EG O-H stretch
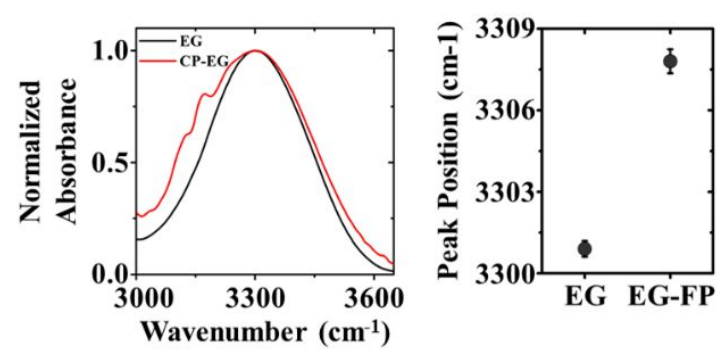

(d)
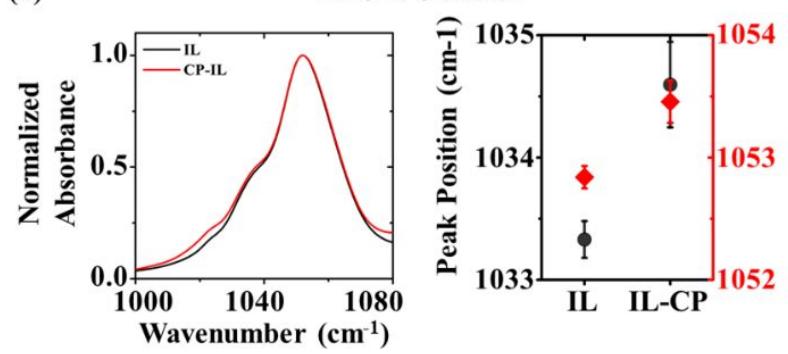

Figure S5. ATR-FTIR spectroscopy of DPP-BTz and templates mixtures. Absorbance spectra and corresponding peak shifts of (a) $\mathrm{FP} \mathrm{CF}_{2}$ stretch, (b) EG O-H stretch, (c) $\mathrm{C}-\mathrm{H}$ vibration of the imidazolium ring of IL cation and (d) SNS antisymmetric vibration of IL anion. Selected characteristic peaks are chosen to have the least interference from the other components peaks. 


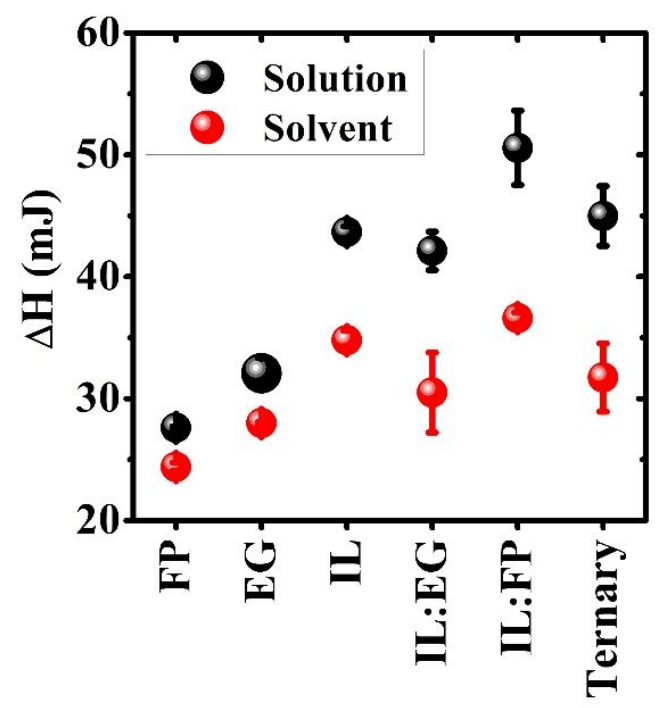

Figure S6. Single-injection isothermal titration calorimetry for quantifying template-CP interactions.

We titrated $3 \mu \mathrm{L}$ of $\mathrm{CP}$ solution in Chloroform or solvent over the templates and measured the released heat per injection. Cumulative enthalpy is obtained by calculating the area under each peak after subtracting the dilution or mechanical effects. At least 3 repeat injections were used to calculate the enthalpy. We obtained the net interaction enthalpy between DPP-BTz and templates from the difference in the heat released at constant pressure during titrating the DPP-BTz solution and the neat solvent (summarized in Figure 7). 